\title{
DEGENERATIONS FOR SELFINJECTIVE ALGEBRAS OF TREECLASS $D_{n}$
}

\author{
ROBERT AEHLE
}

\section{AbSTRACT}

Let $\Lambda$ be a connected representation finite selfinjective algebra. According to G. Zwara the partial orders $\leqslant_{\text {ext }}$ and $\leqslant_{\mathrm{deg}}$ on the isomorphism classes of $d$-dimensional $\Lambda$-modules are equivalent if and only if the stable Auslander-Reiten quiver $\Gamma_{\Lambda}$ of $\Lambda$ is not isomorphic to $\mathbb{Z} D_{3 m} / \tau^{2 m-1}$ for all $m \geqslant 2$. The paper describes all minimal degenerations $M \leqslant_{\operatorname{deg}} N$ with $M K_{\text {ext }} N$ in the case when $\Gamma_{\Lambda} \cong \mathbb{Z} D_{3 m} / \tau^{2 m-1}$ for some $m \geqslant 2$.

\section{Introduction}

\subsection{The affine variety $\bmod _{d} \Lambda$}

Let $k$ be an algebraically closed field and $\Lambda$ be a finite dimensional associative $k$-algebra with unit. We denote by $\bmod \Lambda$ the category of finitely generated $\Lambda$ left-modules. A $d$-dimensional $\Lambda$-module $M$ is the vectorspace $k^{d}$ together with a multiplication by $\Lambda$ from the left.

Now let $\lambda_{1}=1, \lambda_{2}, \ldots, \lambda_{n}$ be a $k$-basis of $\Lambda$. Then $\lambda_{i} \lambda_{j}=\sum_{l} a_{i j}^{l} \lambda_{l}$ for $i, j=1, \ldots, n$ with the structure constants $a_{i j}^{l} \in k$. The multiplication of $M$ by $\lambda_{i}$ induces an endomorphism of $k^{d}$ which we can represent by a $d \times d$ matrix over $k$ with respect to the standard basis of $k^{d}$. Thus $M$ corresponds to a unique $n$-tuple of matrices $m=\left(E, m_{2}, \ldots, m_{n}\right) \in\left(\mathrm{Mat}_{d \times d}(k)\right)^{n}$, where $E$ denotes the identity matrix, and such an $n$-tuple $m$ with $m_{1}=E$ corresponds to a $d$-dimensional $\Lambda$-module if and only if it satisfies the equations $m_{i} m_{j}=\sum_{l} a_{i j}^{l} m_{l}$ for $i, j=1, \ldots, n$. We denote the set of all $n$-tuples corresponding to a $d$-dimensional $\Lambda$-module by $\bmod _{d} \Lambda$ and we will identify the module with its $n$-tuple. For each $i$ with $1 \leqslant i \leqslant n$ let $X^{i}$ denote the matrix $\left(x_{\mu \nu}^{i}\right)_{\mu, v=1, \ldots, d}$. Then $\bmod _{d} \Lambda$ is the zero set of the ideal $I \subset k\left[x_{\mu \nu}^{\xi}\right]$ $(\mu, v=1, \ldots, d ; \xi=1, \ldots, n)$, where $I$ is generated by the components of the matrices $X^{i} X^{j}-\sum_{l} a_{i j}^{l} X^{l}$ for $i, j=1, \ldots, n$. This gives $\bmod _{d} \Lambda$ the structure of an affine variety, which does not have to be irreducible.

The general linear group $\mathrm{Gl}_{d}(k)$ acts on $\bmod _{d} \Lambda$ by conjugation, that is to say $g \cdot\left(m_{1}, \ldots, m_{n}\right)=\left(g m_{1} g^{-1}, \ldots, g m_{n} g^{-1}\right)$ for $g \in \mathrm{Gl}_{d}(k)$ and $\left(m_{1}, \ldots, m_{n}\right) \in \bmod _{d} \Lambda$. The orbits under this action are the isomorphism classes of $d$-dimensional $\Lambda$-modules (see [7]). This definition of $\bmod _{d} \Lambda$ depends on the chosen basis of $\Lambda$ only up to a $\mathrm{Gl}_{d}(k)$ equivariant isomorphism of affine varieties.

\subsection{Partial orders on isomorphism classes of $\bmod _{d} \Lambda$}

A module $N$ is called a degeneration of $M$ (in symbols $M \leqslant_{\operatorname{deg}} N$ ) if $N$ belongs to the Zariski closure of the $\mathrm{Gl}_{d}(k)$-orbit of $M$ in $\bmod _{d} \Lambda$. Since orbits are irreducible

Received 19 February 2001; revised 17 October 2001

2000 Mathematics Subject Classification 14L30, 16G70. 
and open in their closure, this defines a partial order on the set of isomorphism classes of $d$-dimensional $\Lambda$-modules. It is an interesting problem to express the partial order $\leqslant_{\text {deg }}$ in algebraic terms. There are several articles in this direction, including works by S. Abeasis and A. del Fra [1], K. Bongartz [4, 5], C. Riedtmann [11] and G. Zwara $[12,14]$, connecting $\leqslant$ deg to other partial orders on the isomorphism classes of $d$-dimensional $\Lambda$-modules.

In [15] Zwara gives an alternative description of $\leqslant_{\text {deg }}$, that is to say $M \leqslant_{\operatorname{deg}} N$ if and only if there exists a short exact sequence

$$
0 \longrightarrow S \longrightarrow S \oplus M \longrightarrow N \longrightarrow 0
$$

for some $\Lambda$-module $S$.

We are concerned with two other partial orders on the isomorphism classes of $d$-dimensional $\Lambda$-modules. The partial order $\leqslant_{\text {ext }}$ is the transitive closure of the relation $M \leqslant_{\text {ext }} N$ if there exists a short exact sequence

$$
0 \longrightarrow N_{1} \longrightarrow M \longrightarrow N_{2} \longrightarrow 0
$$

with $N \cong N_{1} \oplus N_{2}$. We take the pullback of the sequence (2) with the canonical projection $N \longrightarrow N_{2}$ according to the isomorphism $N \cong N_{1} \oplus N_{2}$. This results in a sequence as in (1) with $S=N_{1}$, so $\leqslant_{\text {ext }}$ implies $\leqslant_{\text {deg }}$.

The hom order $\leqslant$ is the partial order given by $M \leqslant N$ if and only if

$$
[M, X] \leqslant[N, X]
$$

for every $\Lambda$-module $X$, where $[U, V]:=\operatorname{dim}_{k} \operatorname{Hom}_{\Lambda}(U, V)$ for $\Lambda$-modules $U$ and $V$. It follows immediately from (1) and the left-exactness of $\operatorname{Hom}_{\Lambda}(, X)$ that $\leqslant_{\operatorname{deg}}$ implies $\leqslant$. The reverse implication is not true in general. However it holds for representation finite algebras (see [14]) and tame concealed algebras (see [4]).

\subsection{Statement of the theorem}

We define the Auslander-Reiten quiver $\Gamma_{\Lambda}$ of $\Lambda$ as the quiver whose vertices are representatives of the isomorphism classes of indecomposable $\Lambda$-modules. There is an arrow $x \longrightarrow y$ between the vertices $x$ and $y$ if there exists an irreducible morphism from a $\Lambda$-module represented by $x$ to one of $y$. This definition coincides with the usual one in the representation finite case (see [3]) and is appropriate for our consideration.

We denote by $\tau$ the Auslander-Reiten translation. It is a bijection from the isomorphism classes of indecomposable non-projective $\Lambda$-modules to the isomorphism classes of indecomposable non-injective $\Lambda$-modules.

The stable Auslander-Reiten quiver $\Gamma_{\Lambda}^{\mathrm{s}}$ of $\Lambda$ is the full subquiver of $\Gamma_{\Lambda}$ containing all the vertices $x$ for which $\tau^{n}(x)$ is defined for all $n \in \mathbb{Z}$.

Let $\Lambda$ be connected and selfinjective of finite representation type. C. Riedtmann showed in [8] that the stable Auslander-Reiten quiver $\Gamma_{\Lambda}^{\mathrm{s}}$ of $\Lambda$ is isomorphic to $\mathbb{Z} \Delta / G$ where $\Delta$ is one of the Dynkin diagrams $A_{n}, D_{n}, E_{6}, E_{7}, E_{8}$ and $G$ is an admissible automorphism group of $\mathbb{Z} \Delta$. Using the results in [10], [9] and [6] about the category of modules over representation finite selfinjective algebras, G. Zwara showed in [13] that the partial orders $\leqslant_{\text {ext }}$ and $\leqslant_{\text {deg }}$ coincide if and only if $\Gamma_{\Lambda}^{\mathrm{s}} ¥ \mathbb{Z} D_{3 m} / \tau^{2 m-1}$ for all $m \geqslant 2$.

We want to investigate the difference between the partial orders $\leqslant$ deg and $\leqslant_{\text {ext }}$ in those exceptional cases. In particular, we want to describe the minimal degenerations 


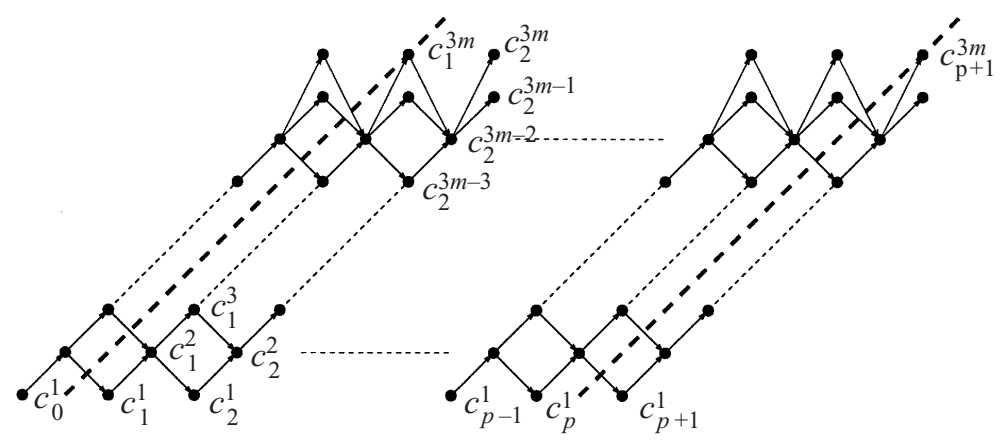

FIGURE 1. $\mathbb{Z} D_{3 m} / \tau^{2 m-1}$.

$M \leqslant_{\operatorname{deg}} N$ with $M \Varangle_{\operatorname{ext}} N$ for a connected selfinjective algebra of finite representation type with stable Auslander-Reiten quiver $\mathbb{Z} D_{3 m} / \tau^{2 m-1}$. A degeneration $M \leqslant_{\operatorname{deg}} N$ is called minimal if it is a proper degeneration, that is to say $M \nsubseteq N$, and if there exists no module $P$ with $M \nsubseteq P \not N$ and $M \leqslant_{\operatorname{deg}} P \leqslant_{\operatorname{deg}} N$. It is an interesting question how complicated minimal degenerations are. Some results concerning the complexity of degenerations can be found in [2].

G. Zwara proved in [15, Theorem 4] that for a minimal degeneration $M \leqslant_{\operatorname{deg}} N$ with $M \Varangle_{\text {ext }} N$ there exist decompositions $M \cong M^{\prime} \oplus W$ and $N \cong N^{\prime} \oplus W$ such that $N^{\prime}$ is indecomposable and $M^{\prime} \leqslant_{\operatorname{deg}} N^{\prime}$ is a minimal degeneration. Therefore it is enough to concentrate on degenerations to indecomposables.

The stable translation quiver $\mathbb{Z} D_{3 m}$ has the vertices $c_{j}^{i}$ where $i \in\{1, \ldots, 3 m\}$ and $j \in \mathbb{Z}$. There are arrows $c_{j}^{i} \longrightarrow c_{j}^{i+1}$ and $c_{j}^{i+1} \longrightarrow c_{j+1}^{i}$ for $1 \leqslant i \leqslant 3 m-2$ and arrows $c_{j}^{3 m-2} \longrightarrow c_{j}^{3 m}$ and $c_{j}^{3 m} \longrightarrow c_{j+1}^{3 m-2}$. The translation is given by $\tau\left(c_{j}^{i}\right)=c_{j-1}^{i}$. Thus the vertices $c_{j}^{i}$ and $c_{j+2 m-1}^{i}$ are identified in the quotient $\mathbb{Z} D_{3 m} / \tau^{2 m-1}$. In Figure 1 the stable Auslander-Reiten quiver $\mathbb{Z} D_{3 m} / \tau^{2 m-1}$ is drawn for $m$ even. Every letter refers to the vertex at its left and the thick diagonal lines indicate the $(2 m-1)$-period of the translation $\tau$.

THEOREM 1.1. Let $\Lambda$ be a connected and selfinjective algebra of finite representation type whose stable Auslander-Reiten quiver is isomorphic to $\mathbb{Z} D_{3 m} / \tau^{2 m-1}$. There exists a proper degeneration $M \leqslant_{\operatorname{deg}} N$ to the indecomposable $\Lambda$-module $N$ if and only if $N$ corresponds to a vertex $c_{l}^{s}$ with $m+1 \leqslant s \leqslant 2 m$. Moreover, the module $M$ is determined by $N$ up to isomorphism.

\section{Preliminaries}

We want to represent pairs of modules $(M, N)$ in terms of $\mathbb{Z}$-valued differencefunctions on the set of isomorphism classes of indecomposable modules and to characterize those functions corresponding to pairs $(M, N)$ with $M \leqslant_{\operatorname{deg}} N$. This will enable us to give a combinatorial proof of the theorem.

We say that the modules $M$ and $N$ are disjoint if they have no common direct summand. We denote by $\bar{M}$ the isomorphism class of the module $M$ and by $\mathscr{S}$ the set of ordered pairs $(\bar{M}, \bar{N})$ such that $M$ and $N$ are disjoint. To every pair $(\bar{M}, \bar{N})$ we associate the function $\delta_{M, N}$ given by $\delta_{M, N}(X)=[N, X]-[M, X]$.

Let $\mu(X, A)$ be the multiplicity of the indecomposable direct summand $X$ in the direct sum decomposition of $A$. In particular $A \cong \bigoplus_{\bar{X} ; X \text { indec }} X^{\mu(X, A)}$. 


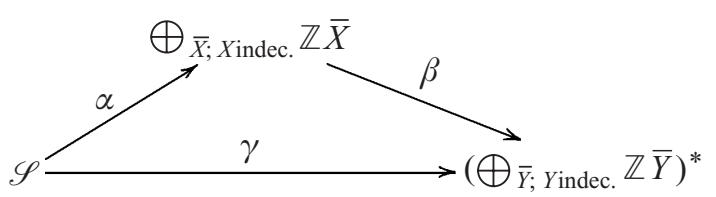

FIGURE 2.

Let $\left(\bigoplus_{\bar{Y} ; Y \text { indec }} \mathbb{Z} \bar{Y}\right)^{*}=\operatorname{Hom}_{\mathbb{Z}}\left(\bigoplus_{\bar{Y} ; Y \text { indec }} \mathbb{Z} \bar{Y}, \mathbb{Z}\right)$. We consider the diagram in Figure 2 where $\alpha, \beta$ and $\gamma$ are given by

$$
\begin{aligned}
\alpha(\bar{M}, \bar{N}) & =\sum_{\bar{X} ; X \text { indec }}(\mu(X, N)-\mu(X, M)) \bar{X}, \\
\gamma(\bar{M}, \bar{N}) & =\delta_{M, N}, \\
\beta\left(\sum_{\bar{X} ; X \text { indec }} \lambda_{\bar{X}} \bar{X}\right) & =\sum_{\bar{X} ; X \text { indec }} \lambda_{\bar{X}}[X, \quad] .
\end{aligned}
$$

The diagram commutes since

$$
\beta \circ \alpha(\bar{M}, \bar{N})=\sum_{\bar{X}}(\mu(X, N)-\mu(X, M))[X, \quad]=[N, \quad]-[M, \quad]=\delta_{M, N} .
$$

Obviously $\alpha$ is a bijection and $\beta$ is $\mathbb{Z}$-linear.

Lemma 2.1. If $\Lambda$ is of finite representation type then $\beta$ is an isomorphism.

Proof. The map $\beta$ is $\mathbb{Z}$-linear between free $\mathbb{Z}$-modules of the same finite rank. Thus it suffices to show that $\beta$ is surjective. For each indecomposable module $X$ we consider the exact sequence

$$
X \longrightarrow E_{X}^{\prime} \longrightarrow \tau^{-1} X \longrightarrow 0
$$

which is the Auslander-Reiten sequence starting in $X$ if $X$ is not injective. Otherwise we set $E_{X}^{\prime}=X / \operatorname{soc}(X)$ and $\tau^{-1} X=0$. The functor $\operatorname{Hom}_{\Lambda}(, Y)$ induces the exact sequence

$$
0 \longrightarrow \operatorname{Hom}_{\Lambda}\left(\tau^{-1} X, Y\right) \longrightarrow \operatorname{Hom}_{\Lambda}\left(E_{X}^{\prime}, Y\right) \longrightarrow \operatorname{Hom}_{\Lambda}(X, Y) \longrightarrow k^{\mu(X, Y)} \longrightarrow 0
$$

of $k$-vectorspaces. Thus for every indecomposable module $Y$ we have

$$
\left([X, \quad]+\left[\tau^{-1} X, \quad\right]-\left[E_{X}^{\prime}, \quad\right]\right)(Y)= \begin{cases}1 & \text { if } Y \cong X \\ 0 & \text { otherwise }\end{cases}
$$

showing that $\beta$ is surjective.

We want to describe the inverse of $\beta$. For each indecomposable module $X$ we consider the exact sequence

$$
0 \longrightarrow \tau X \longrightarrow E_{X} \longrightarrow X
$$

which is the Auslander-Reiten sequence ending in $X$ if $X$ is not projective. Otherwise we set $E_{X}=\operatorname{rad}(X)$ and $\tau X=0$. Then $\beta^{-1}$ is given by

$$
\beta^{-1}(\delta)=\sum_{\bar{X} ; X \text { indec }}\left(\delta(X)+\delta(\tau X)-\delta\left(E_{X}\right)\right) \bar{X} .
$$


Thus, if $\Lambda$ is of finite representation type, $\gamma$ is bijective and we write $\gamma^{-1}(\delta)=$ $\left(\bar{M}_{\delta}, \bar{N}_{\delta}\right) \in \mathscr{S}$. Then

$$
\begin{aligned}
\sum_{\bar{X} ; X \text { indec }}\left(\delta(X)+\delta(\tau X)-\delta\left(E_{X}\right)\right) \bar{X} & =\beta^{-1}(\delta)=\alpha \circ \gamma^{-1}(\delta) \\
& =\sum_{\bar{X} ; X \text { indec }}\left(\mu\left(X, N_{\delta}\right)-\mu\left(X, M_{\delta}\right)\right) \bar{X}
\end{aligned}
$$

and in consequence

$$
\delta(X)+\delta(\tau X)-\delta\left(E_{X}\right)=\mu\left(X, N_{\delta}\right)-\mu\left(X, M_{\delta}\right)
$$

for every indecomposable module $X$ and every $\delta \in\left(\bigoplus_{\bar{Y} ; Y \text { indec }} \mathbb{Z} \bar{Y}\right)^{*}$.

Let $\mathscr{S}^{\prime} \subset \mathscr{S}$ be the subset containing all pairs $(\bar{M}, \bar{N})$ with $M \leqslant_{\operatorname{deg}} N$.

LEMma 2.2. If $\Lambda$ is of finite representation type then $\gamma$ restricts to a bijection between $\mathscr{S}^{\prime}$ and the set of non-negative functions $\delta \in\left(\bigoplus_{\bar{Y} ; Y \text { indec }} \mathbb{Z} \bar{Y}\right)^{*}$ such that $\delta(I)=0$ for every injective module $I$.

Proof. Zwara showed in [14] that the partial orders $\leqslant_{\text {deg }}$ and $\leqslant$ coincide for representation finite algebras. Hence $\gamma(\bar{M}, \bar{N})$ is a non-negative function for every $(\bar{M}, \bar{N}) \in \mathscr{S}^{\prime}$. If $I$ is an injective module then $[N, I]=[M, I]$ holds in consequence of the exactness of $\operatorname{Hom}_{\Lambda}(, I)$ and (1). On the other hand, let $\delta \in\left(\bigoplus_{\bar{Y} ; Y \text { indec }} \mathbb{Z} \bar{Y}\right)^{*}$ be non-negative such that $\delta(I)=0$ for every injective module. We have to show that $\operatorname{dim}_{k} N_{\delta}=\operatorname{dim}_{k} M_{\delta}$ holds. We consider the injective module $\operatorname{Hom}_{k}\left(\Lambda_{\Lambda}, k\right)$, where $\Lambda_{\Lambda}$ denotes $\Lambda$ as $\Lambda$-right module. Then the adjoint isomorphism gives $\left[A, \operatorname{Hom}_{k}\left(\Lambda_{\Lambda}, k\right)\right]=$ $\operatorname{dim}_{k} \operatorname{Hom}_{k}(A, k)=\operatorname{dim}_{k} A$ for every $\Lambda$-module $A$. In particular $\operatorname{dim}_{k} N_{\delta}=\operatorname{dim}_{k} M_{\delta}$.

Let $\mathscr{S}^{\prime}{ }_{N}=\left\{(\bar{X}, \bar{Y}) \in \mathscr{S}^{\prime} \mid \bar{Y}=\bar{N}\right\}$. As a consequence of Lemma 2.2 and (3) we can describe all isomorphism classes of modules degenerating to an indecomposable.

LEMMA 2.3. Let $\Lambda$ be of finite representation type and $N$ be indecomposable. Then $\mathscr{S}^{\prime}{ }_{N}$ is mapped bijectively by $\gamma$ to the set of non-negative functions $\delta \in\left(\bigoplus_{\bar{Y} ; Y \text { indec }} \mathbb{Z} \bar{Y}\right)^{*}$ with $\delta(I)=0$ for every injective module $I$, and satisfying

for every indecomposable $X$.

$$
\delta(X)+\delta(\tau X)-\delta\left(E_{X}\right) \begin{cases}=1 & \text { if } X \cong N \\ \leqslant 0 & \text { otherwise }\end{cases}
$$

Note that for $(\bar{M}, \bar{N})=\gamma^{-1}(\delta)$ we have then

$$
M \cong \bigoplus_{\bar{X} ; X \nsucceq N} X^{-\left(\delta(X)+\delta(\tau X)-\delta\left(E_{X}\right)\right)},
$$

where the direct sum is taken over all isomorphism classes of indecomposable $\Lambda$-modules except that of $N$.

\section{Proof of Theorem 1.1}

Let $\Lambda$ be a selfinjective finite dimensional $k$-algebra of finite representation type with stable Auslander-Reiten quiver isomorphic to $\mathbb{Z} D_{3 m} / \tau^{2 m-1}$. If $M \leqslant_{\operatorname{deg}} N$ is a 
proper degeneration to the indecomposable $N$, then $M$ and $N$ are disjoint. If $N$ were projective then the sequence (1) would split in contradiction to $M \nsubseteq N$. Thus $N$ corresponds to a vertex $c_{l}^{s}$ in the stable Auslander-Reiten quiver of $\Lambda$.

In subsection 3.1 we will characterize a function $\delta \in \gamma\left(\mathscr{S}_{N}^{\prime}\right)$ describing a proper degeneration to $N$ as the unique solution of a linear system depending on two vertices. One of these vertices is the vertex corresponding to $N$. In Subsection 3.2 we analyse this linear system. We will show that if this linear system has a solution in the natural numbers, then this solution is uniquely determined by the vertex $c_{l}^{s}$ and $m+1 \leqslant s \leqslant 2 m$ holds. It follows then from Lemma 2.3 that there exists up to isomorphism at most one module $M$ degenerating to $N$. Finally we give, for the indecomposable module $N$ corresponding to the vertex $c_{l}^{s}$ with $m+1 \leqslant s \leqslant 2 m$, a non-negative function $\delta$ which satisfies (4) for every indecomposable. Thus by Lemma 2.3 there exists a proper degeneration $M \leqslant_{\operatorname{deg}} N$.

By reindexing the stable Auslander-Reiten quiver $\mathbb{Z} D_{3 m} / \tau^{2 m-1}$ we can assume that $l=1$. From now on $N$ always corresponds to the vertex $c_{1}^{s}$.

\subsection{Characterization of $\delta$ by a linear system}

We denote by $p:=2 m-1$ the period of the Auslander-Reiten translation $\tau$ and by $h:=3 m-1$ the 'height' of the Dynkin diagram $D_{3 m}$.

Let us fix an element $\delta \in \gamma\left(\mathscr{S}_{N}^{\prime}\right)$. We set

$$
a_{j}^{i}:= \begin{cases}0 & \text { if } i=0 \\ \delta\left(c_{j}^{i}\right) & \text { if } 1 \leqslant i \leqslant h-1 \\ b_{j}^{h}+b_{j}^{h+1} & \text { if } i=h,\end{cases}
$$

where $b_{j}^{i}:=\delta\left(c_{j}^{i}\right)$ for $h \leqslant i \leqslant h+1$.

Note that all the integers $a_{j}^{i}$ and $b_{j}^{i}$ are non-negative. We consider for each vertex $c_{j}^{i}$ the Auslander-Reiten sequence ending in $c_{j}^{i}$. Since $\delta(I)=0$ if $I$ is an injective $\Lambda$-module we obtain the following set of inequalities from Lemma 2.3.

If $1 \leqslant i \leqslant h-1$

$$
a_{j}^{i}+a_{j-1}^{i}-a_{j-1}^{i+1}-a_{j}^{i-1} \begin{cases}=1 & \text { if } c_{j}^{i}=c_{1}^{s} \\ \leqslant 0 & \text { otherwise. }\end{cases}
$$

If $h \leqslant i \leqslant h+1$

$$
b_{j}^{i}+b_{j-1}^{i}-a_{j}^{h-1} \begin{cases}=1 & \text { if } c_{j}^{i}=c_{l}^{s} \\ \leqslant 0 & \text { otherwise. }\end{cases}
$$

These inequalities are the key to proving the theorem. First we derive some information on the $\tau$-orbits in $\mathbb{Z} D_{3 m} / \tau^{2 m-1}$. We sum up the $\delta$-values along each $\tau$-orbit and set

$$
\begin{aligned}
& a_{i}=\sum_{j=1}^{p} a_{j}^{i} \quad \text { for } 0 \leqslant i \leqslant h, \\
& b_{i}=\sum_{j=1}^{p} b_{j}^{i} \quad \text { for } h \leqslant i \leqslant h+1 .
\end{aligned}
$$


Then by definition $a_{h}=b_{h}+b_{h+1}$ and $a_{0}=0$. For each fixed $i$ we add up the inequalities of (6) and (7) respectively and we obtain

$$
\begin{array}{ll}
2 a_{i} \leqslant a_{i+1}+a_{i-1}+\delta_{i, s} & \text { for } 1 \leqslant i \leqslant h-1, \\
2 b_{i} \leqslant a_{h-1}+\delta_{i, s} & \text { for } h \leqslant i \leqslant h+1 .
\end{array}
$$

Here $\delta_{i, s}$ denotes the Kronecker symbol. By definition and the inequality (9) we get $2 a_{h}=2 b_{h}+2 b_{h+1} \leqslant 2 a_{h-1}+1$. Hence

$$
a_{h} \leqslant a_{h-1} .
$$

REMARK 3.1. From $\delta(N)+\delta(\tau N)-\delta\left(E_{N}\right)=1$ it follows immediately that $a_{s} \geqslant$ $\delta(N)+\delta(\tau N)>0$ if $s \leqslant h-1$ and $a_{h}>0$ if $s \geqslant h$.

The following lemma implies that the case $s \geqslant h$ does not occur. In view of Figure 1 this means that the vertex $c_{1}^{s}$ is not one of the somehow exceptional vertices on the upper boundary.

LEMma 3.2. It holds that $s \leqslant h-1$ and there exists an integer $t$ with $2 \leqslant t \leqslant s$ such that

$$
t=2 b_{h}=2 b_{h+1} \quad \text { and } \quad a_{i}= \begin{cases}0 & \text { if } 0 \leqslant i \leqslant s-t \\ i-(s-t) & \text { if } s-t \leqslant i \leqslant s \\ t & \text { if } s \leqslant i \leqslant h .\end{cases}
$$

In particular $a_{s}=t$ is an even integer.

Proof. The inequalities in (8) are equivalent to

$$
a_{i}-a_{i-1} \leqslant a_{i+1}-a_{i}+\delta_{i, s}
$$

for $1 \leqslant i \leqslant h-1$. Suppose that $s \geqslant h$. It follows from (11) and (10) that

$$
0 \leqslant a_{1}=a_{1}-a_{0} \leqslant \ldots \leqslant a_{h}-a_{h-1} \leqslant 0 .
$$

This implies that $a_{i}=0$ for all $i \in\{1, \ldots, h\}$ in contradiction to $a_{h}>0$ by Remark 3.1. Hence $s \leqslant h-1$.

Again by (11) and (10) we obtain the following chain of inequalities:

$$
\begin{aligned}
0 \leqslant a_{1} & =a_{1}-a_{0} \leqslant a_{2}-a_{1} \leqslant \ldots \leqslant a_{s}-a_{s-1} \\
& \leqslant a_{s+1}-a_{s}+1 \leqslant \ldots \leqslant a_{h}-a_{h-1}+1 \leqslant 1 .
\end{aligned}
$$

If $a_{s}-a_{s-1}=0$ then $a_{1}=a_{2}=\ldots=a_{s}=0$ in contradiction to $a_{s}>0$ by Remark 3.1. Hence there is an integer $t$ with $0<t \leqslant s$ such that

$$
\begin{aligned}
0 & =a_{1}-a_{0}=\ldots=a_{s-t}-a_{s-t-1}, \\
1 & =a_{s-t+1}-a_{s-t}=\ldots=a_{s}-a_{s-1} \\
& =a_{s+1}-a_{s}+1=\ldots=a_{h}-a_{h-1}+1 .
\end{aligned}
$$

Our claim for the $a_{i}$ is an easy consequence. In particular we have $a_{h-1}=a_{h}=$ $b_{h}+b_{h+1}$, but $2 b_{h+1} \leqslant a_{h-1}$ and $2 b_{h} \leqslant a_{h-1}$ by (9). Hence we see that $t=a_{h-1}=$ $2 b_{h}=2 b_{h+1}$ is an even integer. 
As an immediate consequence of Lemma 3.2 we note that

$$
2 a_{i}-a_{i+1}-a_{i-1}= \begin{cases}1 & \text { if } i=s \\ -1 & \text { if } i=s-t \\ 0 & \text { otherwise }\end{cases}
$$

for $1 \leqslant i \leqslant h-1$.

We want to describe the non-negative integers $a_{j}^{i}$ and $b_{j}^{i}$ and hence the function $\delta$ as the unique solution of a linear system. By Lemma 3.2 we have $1=a_{s-t+1}=$ $\sum_{j} \delta\left(c_{j}^{s-t+1}\right)$, so there exists exactly one vertex $c_{u-1}^{s-t+1}$ with $\delta\left(c_{u-1}^{s-t+1}\right)=a_{u-1}^{s-t+1}=1$. Note that the index $u$ is only determined modulo $p$. In the sequel let $\tilde{u}$ be the representative of $u$ with $1 \leqslant \tilde{u} \leqslant p$.

If $s>t$ the Auslander-Reiten sequence ending in $c_{u}^{s-t}$ gives rise to the equation

$$
a_{u}^{s-t}+a_{u-1}^{s-t}-a_{u-1}^{s-t+1}-a_{u}^{s-t-1}=-1
$$

because $a_{i}=0$ for $i \leqslant s-t$, by Lemma 3.2.

We consider the following linear system which depends on the positions of the vertices $c_{1}^{s}$ and $c_{u-1}^{s-t+1}$ or equivalently on the integers $s, t$ and $\tilde{u}$. The lower index is taken to be in $\mathbb{Z} / p \mathbb{Z}$.

$$
x_{j}^{0}=0, \quad x_{j}^{1}=\left\{\begin{array}{ll}
1 & \text { if } c_{j}^{1}=c_{u-1}^{s-t+1} \\
0 & \text { otherwise, }
\end{array} \quad x_{j}^{h}=y_{j}^{h}+y_{j}^{h+1} .\right.
$$

If $1 \leqslant i \leqslant h-1$

$$
x_{j}^{i}+x_{j-1}^{i}-x_{j-1}^{i+1}-x_{j}^{i-1}= \begin{cases}1 & \text { if } c_{j}^{i}=c_{1}^{s} \\ -1 & \text { if } c_{j}^{i}=c_{u}^{s-t} \\ 0 & \text { otherwise. }\end{cases}
$$

If $h \leqslant i \leqslant h+1$

$$
y_{j}^{i}+y_{j-1}^{i}=x_{j}^{h-1} .
$$

Lemma 3.3. If $x_{j}^{i}, y_{j}^{i}$ is a rational solution of the linear system (14)-(16) then $y_{j}^{h+1}=y_{j}^{h}$ holds for all $j$.

Proof. Suppose that there is $j_{0}$ with $y_{j_{0}}^{h+1}>y_{j_{0}}^{h}$. Since $y_{j}^{h+1}+y_{j+1}^{h+1}=x_{j+1}^{h-1}=$ $y_{j}^{h}+y_{j+1}^{h}$ by (16) we have $-y_{j_{0}+1}^{h+1}>-y_{j_{0}+1}^{h}$ and successively

$$
-y_{j_{0}}^{h+1}=(-1)^{p} y_{j_{0}+p}^{h+1}>(-1)^{p} y_{j_{0}+p}^{h}=-y_{j_{0}}^{h}, \quad \text { as } p \text { is odd, }
$$

in contradiction to $y_{j_{0}}^{h+1}>y_{j_{0}}^{h}$.

To any integer solution $x_{j}^{i}, y_{j}^{i}$ of this linear system we can associate a function $\delta^{\prime} \in\left(\bigoplus_{\bar{Y} ; Y \text { indec }} \mathbb{Z} \bar{Y}\right)^{*}$ by setting $\delta^{\prime}\left(c_{j}^{i}\right)=x_{j}^{i}$ for $1 \leqslant i \leqslant h-1, \delta^{\prime}\left(c_{j}^{i}\right)=y_{j}^{i}$ for $h \leqslant i \leqslant h+1$ and $\delta^{\prime}(I)=0$ for every injective module $I$. Under the same conditions we will speak of a function $\delta^{\prime} \in\left(\bigoplus_{\bar{Y} ; Y \text { indec }} \mathbb{Z} \bar{Y}\right)^{*}$ as a solution of the linear system (14)-(16). 
LEMMA 3.4. The unique solution of the linear system (14)-(16) is given by $x_{j}^{i}=a_{j}^{i}$ and $y_{j}^{i}=b_{j}^{i}$. In particular $a_{j}^{h}=2 b_{j}^{h}$ is an even integer for all $j$.

Proof. With respect to uniqueness, it is obvious that the values of $x_{j}^{i}$ are determined by the equations in (14) and (15). The values $y_{j}^{i}$ are given by $y_{j}^{i}=x_{j}^{h} / 2$ by (14) and Lemma 3.3.

The equations in (14) are obviously satisfied by $a_{j}^{i}$ and $b_{j}^{i}$.

Because of (13) and $a_{1}^{s}+a_{0}^{s}-a_{0}^{s+1}-a_{1}^{s-1}=1$ it remains for (15) to show that

$$
a_{j}^{i}+a_{j-1}^{i}-a_{j-1}^{i+1}-a_{j}^{i-1}=0
$$

for $c_{u}^{s-t} \neq c_{j}^{i} \neq c_{1}^{s}$. By (12) we have

$$
0=2 a_{i}-a_{i+1}-a_{i-1}=\sum_{j}\left(a_{j}^{i}+a_{j-1}^{i}-a_{j-1}^{i+1}-a_{j}^{i-1}\right)
$$

for $s \neq i \neq s-t$ and

$$
0=2 a_{s-t}-a_{s-t+1}-a_{s-t-1}+1=\sum_{j \neq u}\left(a_{j}^{s-t}+a_{j-1}^{s-t}-a_{j-1}^{s-t+1}-a_{j}^{s-t-1}\right)
$$

for $i=s-t$ and

$$
0=2 a_{s}-a_{s+1}-a_{s-1}-1=\sum_{j \neq 1}\left(a_{j}^{s}+a_{j-1}^{s}-a_{j-1}^{s+1}-a_{j}^{s-1}\right)
$$

for $i=s$. However because of (6) each of the summands on the right-hand side is less than or equal to zero. Thus each summand on the right-hand side is zero.

Concerning (16) we remark that for $h \leqslant i \leqslant h+1$ we have

$$
0=2 b_{i}-a_{h-1}=\sum_{j}\left(b_{j}^{i}+b_{j-2}^{i}-a_{j-1}^{h-1}\right)
$$

by Lemma 3.2. Since each summand on the right-hand side is less than or equal to zero by (7) it is zero.

Finally we have $a_{j}^{h}=2 b_{j}^{h}$ by (14) and Lemma 3.3 .

This means that every $\delta \in \gamma\left(\mathscr{S}_{N}^{\prime}\right)$ is the unique solution of a linear system which itself depends on some vertex $c_{u-1}^{s-t+1}$. In the next section we will show that $c_{u-1}^{s-t+1}$ is uniquely determined by $c_{1}^{s}$.

\subsection{Computation of $c_{u-1}^{s-t+1}$}

Note that the vertices $c_{1}^{s}$ and $c_{u-1}^{s-t+1}$ have to be positioned in $\mathbb{Z} D_{3 m} / \tau^{2 m-1}$ in such a way that the unique solution of the linear system (14)-(16) takes values in $\mathbb{N}$. We will show that this condition implies that $m+1 \leqslant s \leqslant 2 m$ and determines $c_{u-1}^{s-t+1}$ as $c_{s-m}^{2 m-s+1}$.

It is possible to solve this problem directly by examining the linear system (14) (16), but this procedure is rather complicated. Therefore we use a different method. We will ignore the equations of (16) and only use that $a_{h}^{j}$ is even. Furthermore we use a covering technique to simplify the computations.

To this purpose we consider the stable translation-quiver $\mathbb{Z} A_{h}$ with vertices $d_{j}^{i}$ where $1 \leqslant i \leqslant h$ and $j \in \mathbb{Z}$. There are arrows from $d_{j}^{i}$ to $d_{j}^{i+1}$ and from $d_{j}^{i+1}$ to $d_{j+1}^{i}$ for $1 \leqslant i \leqslant h-1$. The translation $\tau$ is given by $\tau\left(d_{j}^{i}\right)=d_{j-1}^{i}$. In particular we are interested in two quotients of $\mathbb{Z} A_{h}$, namely $Q_{1}:=\mathbb{Z} A_{h} / \tau^{p}$ and $Q_{2}:=\mathbb{Z} A_{h} / \tau^{2 p}$. 


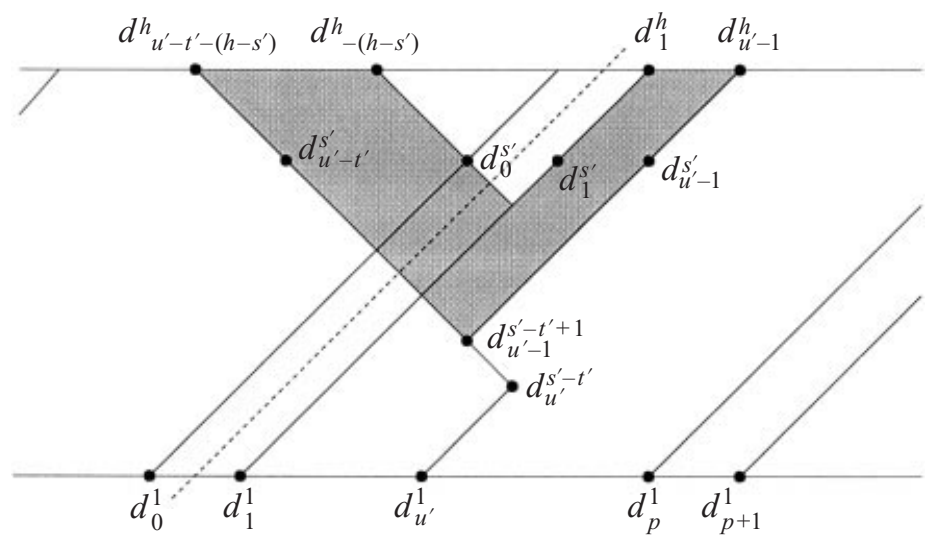

FIGURE 3. $Q_{2}$.

Now let $s^{\prime}, t^{\prime}, u^{\prime} \in \mathbb{N}$ with $2 \leqslant t^{\prime} \leqslant s^{\prime} \leqslant h-1$ and $1 \leqslant u^{\prime} \leqslant p$. Let $\delta_{r}$ : $\left\{\right.$ vertices of $\left.Q_{r}\right\} \longrightarrow \mathbb{Z}$ for $r=1,2$ be a function satisfying

$$
\begin{aligned}
& \delta_{r}\left(d_{j}^{1}\right)= \begin{cases}1 & \text { if } d_{j}^{1}=d_{u^{\prime}-1}^{s^{\prime}-t^{\prime}+1} \\
0 & \text { otherwise, },\end{cases} \\
& \delta_{r}\left(d_{j}^{1}\right)+\delta_{r}\left(d_{j-1}^{1}\right)-\delta_{r}\left(d_{j-1}^{2}\right)= \begin{cases}-1 & \text { if } d_{j}^{1}=d_{u^{\prime}}^{s^{\prime}-t^{\prime}} \\
0 & \text { otherwise, }\end{cases} \\
& \delta_{r}\left(d_{j}^{i}\right)+\delta_{r}\left(d_{j-1}^{i}\right)-\delta_{r}\left(d_{j-1}^{i+1}\right)-\delta_{r}\left(d_{j}^{i-1}\right)= \begin{cases}1 & \text { if } d_{j}^{i}=d_{1}^{s^{\prime}} \\
-1 & \text { if } d_{j}^{i}=d_{u^{\prime}}^{s^{\prime}-t^{\prime}} \\
0 & \text { otherwise, },\end{cases}
\end{aligned}
$$

for $2 \leqslant i \leqslant h-1$.

The values of $\delta_{r}\left(d_{j}^{1}\right)$ are determined by condition (17). The values of $\delta_{r}\left(d_{j}^{i}\right)$ with $i \geqslant 2$ are determined by the values of $\delta_{r}\left(d_{j}^{i^{\prime}}\right)$ with $i^{\prime}<i$ because of conditions (18) and (19). Thus there exists exactly one such function $\delta_{r}$.

The function $\delta_{2}$ is easy to calculate.

Lemma 3.5. If $u^{\prime}-t^{\prime} \leqslant 1$ then the function $\delta_{2}$ is given by $\delta_{2}\left(d_{j}^{i}\right)=1$ if the vertex $d_{j}^{i}$ lies in the shaded area (including the boundary) of Figure 3 and $\delta_{2}\left(d_{j}^{i}\right)=0$ otherwise.

Proof. It is straightforward to check that Equations (17), (18) and (19) hold.

We define the function $\delta_{1}^{\prime}:\left\{\right.$ vertices of $\left.Q_{1}\right\} \longrightarrow \mathbb{Z}$ by $\delta_{1}^{\prime}\left(d_{j}^{i}\right)=\delta_{2}\left(d_{j}^{i}\right)+\delta_{2}\left(d_{j+p}^{i}\right)$. Of course $\delta_{1}^{\prime}$ satisfies Equations (17), (18) and (19), from which we see that $\delta_{1}^{\prime}=\delta_{1}$ and consequently

$$
\delta_{1}\left(d_{j}^{i}\right)=\delta_{2}\left(d_{j}^{i}\right)+\delta_{2}\left(d_{j+p}^{i}\right)
$$

We note the following.

Lemma 3.6. If $u^{\prime}-t^{\prime} \leqslant 1$ then $\delta_{1}\left(d_{j}^{i}\right)$ is an even integer if and only if $\delta_{2}\left(d_{j}^{i}\right)=$ $\delta_{2}\left(d_{j+p}^{i}\right)$. 
Proof. Since $\delta_{2}$ takes only values in $\{0,1\}$ by Lemma 3.5 the claim follows from (20).

Let us now consider again our function $\delta \in \gamma\left(\mathscr{S}_{N}{ }_{N}\right)$. This function induces a function $\bar{\delta}:\left\{\right.$ vertices of $\left.Q_{1}\right\} \longrightarrow \mathbb{Z}$ by $\bar{\delta}\left(d_{j}^{i}\right)=a_{j}^{i}$. We set $s^{\prime}=s, t^{\prime}=t$ and $u^{\prime}=\bar{u}$.

Then the function $\bar{\delta}$ satisfies Equations (17), (18) and (19) by Lemma 3.4 and consequently $\bar{\delta}=\delta_{1}$.

Lemma 3.7. If $\delta \in \gamma\left(\mathscr{S}_{N}^{\prime}\right)$ is the unique solution of the linear system (14)-(16) then we have $\tilde{u}-t \leqslant 1$.

Proof. Suppose the lemma is false. Then $1<\tilde{u}-t \leqslant \tilde{u} \leqslant p$ and in consequence none of the integers $2 p, 1, p, p+1$ is congruent modulo $2 p$ to an integer in $\{\tilde{u}-t$, $\tilde{u}-t+1, \ldots, \tilde{u}-1\}$. By (17)-(19) and since $2 \leqslant t<p$ we have

$$
\delta_{2}\left(d_{j}^{s}\right)= \begin{cases}1 & \text { if } d_{j}^{s}=d_{j^{\prime}}^{s} \text { with } j^{\prime} \in\{\tilde{u}-t, \tilde{u}-t+1, \ldots, \tilde{u}-1\} \\ 0 & \text { otherwise. }\end{cases}
$$

Hence by (20) and Remark 3.1

$$
\begin{aligned}
0 & =\delta_{2}\left(d_{1}^{s}\right)+\delta_{2}\left(d_{1+p}^{s}\right)+\delta_{2}\left(d_{2 p}^{s}\right)+\delta_{2}\left(d_{p}^{s}\right) \\
& =\bar{\delta}\left(d_{1}^{s}\right)+\bar{\delta}\left(d_{p}^{s}\right) \\
& =\delta(N)+\delta(\tau N) \\
& \geqslant 1
\end{aligned}
$$

which is obviously a contradiction.

We are able now to determine $\tilde{u}$ and $t$ by means of $s$.

Lemma 3.8. If $\delta \in \gamma\left(\mathscr{S}_{N}^{\prime}\right)$ solves the linear system (14)-(16) then $c_{u-1}^{s-t+1}=c_{s-m}^{2 m-s+1}$ and $m+1 \leqslant s \leqslant 2 m$ holds. In particular $t=2(s-m)$ and $\tilde{u}=t / 2+1$.

Proof. By Lemma 3.7 we have $\tilde{u}-t \leqslant 1$. Therefore we can apply Lemma 3.5 to describe $\delta_{2}$. On the other hand, we know from Lemma 3.4 that $\delta_{1}\left(d_{j}^{h}\right)=\bar{\delta}\left(d_{j}^{h}\right)=a_{j}^{h}$ is always an even integer. In view of Lemma 3.6 this means that $\delta_{2}\left(d_{j}^{h}\right)=\delta_{2}\left(d_{j+p}^{h}\right)$ for all $j$. Thus we have (see Figure 3)

$$
\begin{aligned}
\tilde{u}-1-1 & \equiv-(h-s)-(\tilde{u}-t-(h-s)) \bmod (2 p), \\
-(h-s)+p & \equiv \tilde{u}-1 \bmod (2 p) .
\end{aligned}
$$

Substituting $p$ by $2 m-1$ and $h$ by $3 m-1$ we calculate that $\tilde{u}=t / 2+1$ and $t=2(s-m)$. Since $2 \leqslant t \leqslant s$ by Lemma 3.2 we get $2 \leqslant 2(s-m) \leqslant s$ which is equivalent to $m+1 \leqslant s \leqslant 2 m$.

Hence the existence of a proper degeneration to the indecomposable $N$ corresponding to the vertex $c_{1}^{s}$ implies that $m+1 \leqslant s \leqslant 2 m$. Furthermore, if $M \leqslant_{\operatorname{deg}} N$ is a proper degeneration to $N$, then $\gamma((\bar{M}, \bar{N})) \in\left(\bigoplus_{\bar{Y} ; Y \text { indec }} \mathbb{Z} \bar{Y}\right)^{*}$ is the unique solution of the linear system (14)-(16) with $c_{u-1}^{s-t+1}=c_{s-m}^{2 m-s+1}$, by Lemma 3.4 and Lemma 3.8. Hence there exists up to isomorphism at most one module $M$ degenerating to $N$. 
On the other hand, let $m+1 \leqslant s \leqslant 2 m$ and $N$ correspond to the vertex $c_{1}^{s}$. We set $s^{\prime}=s, t^{\prime}=2(s-m)$ and $u^{\prime}=t^{\prime} / 2+1$ and define $\delta_{2}$ according to (17), (18) and (19). Since $u^{\prime}-t^{\prime} \leqslant 1$ the function $\delta_{2}$ is described by Lemma 3.5. In consequence we have $\delta_{2}\left(d_{j}^{h}\right)=\delta_{2}\left(d_{j+p}^{h}\right)$. We define $\delta$ by

$$
\delta\left(c_{j}^{i}\right)= \begin{cases}\delta_{2}\left(d_{j}^{i}\right)+\delta_{2}\left(d_{j+p}^{i}\right) & \text { if } 1 \leqslant i \leqslant h-1 \\ 1 / 2\left(\delta_{2}\left(d_{j}^{h}\right)+\delta_{2}\left(d_{j+p}^{h}\right)\right) & \text { if } h \leqslant i \leqslant h+1 .\end{cases}
$$

Then $\delta$ takes value in $\mathbb{N}$ and solves the linear system (14)-(16). Indeed (14) is a consequence of (17), (15) of (18) and (19), and (16) can be checked easily using Lemma 3.5. Thus $\delta \in \gamma\left(\mathscr{S}_{N}^{\prime}\right)$ which means in view of Lemma 2.3 that there exists a proper degeneration to $N$. This completes the proof of Theorem 1.1.

Note that the function $\delta$ constructed above for the indecomposable module $N$ corresponding to the vertex $c_{1}^{s}$ describes the module $M$ degenerating to $N$ in the following way. Let $P_{1}, \ldots, P_{m}$ be representatives of the isomorphism classes of projective indecomposable $\Lambda$-modules. By Lemma 3.4 and (5)

$$
M \cong M_{1} \oplus \bigoplus_{i=1}^{m} P_{i}^{\delta\left(\operatorname{rad} P_{i}\right)}
$$

where $M_{1}$ corresponds to the vertex $c_{s-m+1}^{2 m-s}$ if $s<2 m$ and $M_{1}=0$ if $s=2 m$.

Acknowledgements. The results presented in this paper form a part of the author's doctoral dissertation written under the supervision of Professor C. Riedtmann. The author thanks C. Riedtmann and G. Zwara for helpful discussions and gratefully acknowledges support from the Schweizerischer Nationalfonds.

\section{References}

1. S. Abeasis and A. DEL Fra, 'Degenerations for the representations of a quiver of type $A_{m}$ ', J. Algebra 93 (1985) 376-412.

2. R. Aehle, C. Riedtmann and G. Zwara, 'Complexity of degenerations of modules', Comment. Math. Helv. 76 (2001) 781-803.

3. M. Auslander, I. Reiten and S. O. Smalo, Representation theory of Artin algebras, Cambridge Studies in Advanced Mathematics 36 (Cambridge University Press, 1995).

4. K. Bongartz, 'Degenerations for representations of tame quivers', Ann. Sci. École Norm. Sup. 28 (1995) 647-668.

5. K. Bongartz, 'On degenerations and extensions of finite dimensional modules', Adv. Math. 121 (1996) 245-287.

6. O. Bretscher, C. Läser and C. Riedtmann, 'Selfinjective and simply connected algebras', Manuscripta Math. 36 (1981) 253-307.

7. H. KRAFT, 'Geometric methods in representation theory', Representations of algebras, Lecture Notes in Mathematics 944 (Springer, 1982) 180-258.

8. C. Riedtmann, 'Algebren, Darstellungsköcher und zurück', Comment. Math. Helv. 55 (1980) 199-224.

9. C. RiedtmanN, 'Representation-finite selfinjective algebras of class $A_{n}$ ', Representation theory II, Lecture Notes in Mathematics 832 (Springer, 1980) 449-520.

10. C. Riedtmann, 'Representation-finite selfinjective algebras of class $D_{n}$ ', Compositio Math. 49 (1983) 231-282.

11. C. Riedtmann, 'Degenerations for representations of quivers with relations', Ann. Sci. École Norm. Sup. 4 (1986) 275-301.

12. G. ZWARA, 'Degenerations for modules over representation-finite biserial algebras', J. Algebra 198 (1997) 563-581.

13. G. ZwARA, 'Degenerations for modules over representation-finite selfinjective algebras', Colloq. Math. 75 (1998) 91-95. 
14. G. Zwara, 'Degenerations for modules over representation-finite algebras', Proc. Amer. Math. Soc. 127 (1999) 1313-1322.

15. G. ZwARA, 'Degenerations of finite dimensional modules are given by extensions', Compositio Math 121 (2000) 205-218.

Mathematisches Institut

Universität Bern

Sidlerstrasse 5

3012 Bern

Switzerland

aehle@math-stat.unibe.ch 